\title{
GABA-induced Inactivation of Functionally Characterized Sites in Cat Visual Cortex (Area 18): Effects on Orientation Tuning
}

\author{
John M. Crook and Ulf T. Eysel \\ Department of Neurophysiology, Faculty of Medicine, Ruhr University of Bochum, W-4630 Bochum, Germany
}

Microiontophoresis of the inhibitory transmitter GABA was used to reversibly inactivate small sites of defined orientation specificity at a horizontal distance of some $600 \mu \mathrm{m}$ from single cells recorded in area 18 of cat visual cortex, and the effects on orientation tuning were studied. The receptive fields of cells at the recording and inactivation sites overlapped extensively. During the inactivation of sites where the orientation preference differed by $45^{\circ}$ or more from that of a recorded cell ("cross-orientation" sites), $65 \%$ of 54 cells tested showed significant broadening of orientation tuning, with a mean increase in tuning width (measured at half the maximum response) of $93 \%$, and an almost fourfold increase in the relative response to the orientation orthogonal to the optimum, compared with the response to the optimum; four cells essentially lost their orientation tuning. Broadening of tuning reflected an increase in response to nonoptimal orientations and was reversible upon termination of GABA application. The effects on orientation tuning typically peaked within 10-15 min of the onset of GABA iontophoresis with 50-100 nA ejecting currents, and could not be replicated by inactivating sites where the orientation preference was similar to that of a recorded cell; when the orientation preference at the inactivation sites was within $22.5^{\circ}$ of that of a recorded cell ("iso-orientation" sites), only 3 of 22 cells showed significant broadening of tuning, and in these cases, the effects were relatively weak (mean increase in tuning width of $39 \%$ and a negligible change in the relative response to the orientation orthogonal to the optimum). The effect of inactivating "iso-orientation" sites consisted primarily in an increase in response magnitude. The difference in the magnitude of the effects on orientation tuning elicited by inactivating "cross-orlentation" and "iso-orientation" sites was highly statistically significant. Additionally, inactivation of "cross-orientation" or "iso-orientation" sites elicited differential effects on orientation tuning in 10 of the 13 cells in which direct comparisons were made. It is argued that the observed broadening of tuning was due to the loss of a "cross-orientation" inhibitory input, which normally sharp-

\footnotetext{
Received Aug. 7, 1991; revised Dec. 9, 1991; accepted Dec. 12, 1991.

This article is dedicated to the memory of Prof. O. D. (Otto) Creutzfeldt, who was one of the first to appreciate the importance of intracortical inhibition for the response specificity of visual cortical cells.

This work was supported by grants from the European Communities [SC1* 0329-C(MB)] and the Deutsche Forschungsgemeinschaft (EY8/17-1).

Correspondence should be addressed to Dr. J. M. Crook, Department of Neurophysiology, Faculty of Medicine, Ruhr University of Bochum, POB 102148, W-4630 Bochum 1, Germany.

Copyright @ 1992 Society for Neuroscience $0270-6474 / 92 / 121816-10 \$ 05.00 / 0$
}

ens orientation tuning by suppressing responses to nonoptimal orientations.

Since the discovery that cells in cat visual cortex are selective for the orientation of contours (Hubel and Wiesel, 1962), the mechanisms underlying cortical orientation selectivity have been the subject of intense debate (Hubel and Wiesel, 1962, 1965; Creutzfeldt et al., 1974; Sillito, 1979, 1984; Heggelund, 1981; Ferster, 1986; Ferster and Koch, 1987; Martin, 1988). In particular, while a number of studies (Sillito, 1979; Morrone et al., 1982; Ramoa et al., 1986; Hata et al., 1988) have implicated inhibitory connections between cells with different preferred orientations in the generation of orientation selectivity, such "cross-orientation" inhibition has not been detected in a recent intracellular study (Ferster, 1986). We have previously reported broadening of orientation tuning in areas 17 and 18 of cat visual cortex during iontophoretic application of GABA at a series of locations surrounding a single cell recorded at a horizontal distance of some 500-600 $\mu \mathrm{m}$ (Eysel et al., 1990; Crook et al., 1991a). Our interpretation of the results was that a "cross-orientation" inhibitory input to the recorded cell had been inactivated during GABA application, but the crucial evidence concerning the orientation preference at the inactivation sites was lacking. The present study from area 18, in which provision was made for recording from the inactivation sites, specifically addressed this issue. Determination of the orientation specificity at each site, together with verification of reversible inactivation by GABA, allowed a comparison of the effects of inactivating sites where the orientation preference was similar to or radically different from that of a recorded cell.

Some of the results have been presented previously in abstract form (Crook and Eysel, 1991a,b; Crook et al., 1991b).

\section{Materials and Methods}

A full account of physiological preparation and monitoring, visual stimulation, and quantification of orientation tuning has been published (Crook et al., 1991a), and only aspects of methodology specific to the present study are described in detail.

Anesthesia was induced in seven adult cats $(2.5-5.0 \mathrm{~kg})$ with Ketanest, together with Rompun (i.m.), and tracheal and arterial cannulas were inserted. Following fixation in a stereotaxic frame, and upon commencement of arterial infusion of Alloferin for paralysis, anesthesia was maintained by artificial ventilation with a nitrous oxide/oxygen/halothane gas mixture, and the EEG, pulse rate, and arterial blood pressure were monitored continuously as indicators of anesthetic efficacy. Endtidal $\mathrm{CO}_{2}$ was maintained at $3.8-4.0 \%$ and body temperature near $38.5^{\circ} \mathrm{C}$. Following a craniotomy and durotomy, the eyes were treated with phenylephrine hydrochloride and atropine sulfate, and protected with zeropower contact lenses. Supplementary lenses for focal correction (selected retinoscopically) and 5-mm-diameter artificial pupils were installed. All wound margins and pressure points were locally anesthetized. Record- 
ings were made under light anesthesia with $70: 30 \% \mathrm{~N}_{2} \mathrm{O} / \mathrm{O}_{2}$ supplemented continuously with $0.4-0.6 \%$ halothane.

Single units were recorded extracellularly during vertical penetrations (Horsley-Clarke coordinates: A, $0-2 ; \mathrm{L}, 2.5-3.0$ ) with a $3 \mathrm{M} \mathrm{NaCl}$-filled glass microelectrode (tip diameter, 1-2 $\mu \mathrm{m}$; impedance, 1-5 M 2 ) through the center of a fixed array of four double-barrel pipettes (tip diameter, $10-20 \mu \mathrm{m}$ ), each located at a horizontal distance of some $600 \mu \mathrm{m}$, in the superficial layers $(500-800 \mu \mathrm{m})$ of area 18 (see Fig. 1 and Fig. 2, inset). The array was constructed according to the method described in Crook et al. (1991a). One barrel of each pipette in the array contained GABA $(0.5 \mathrm{M}, \mathrm{pH} 3.0)$ for local inactivation, and the other contained $3 \mathrm{M} \mathrm{NaCl}$ to allow recording of multiunit activity. The barrels had a staggered conformation to prevent cross-contamination and were sealed with silicone wax. A $-15 \mathrm{nA}$ retaining current was used to prevent leakage of GABA. After penetration of the cortical surface with the electrode for single-unit recording, the craniotomy was sealed with agar. The receptive field limits and orientation specificity of multiunit clusters recorded at one of the inactivation sites were determined for each monocular input. It was then verified that iontophoretic application of GABA at the same site reversibly abolished visually driven and spontaneous multiunit activity. Without moving the array, this procedure was carried out for each of the other three sites in turn. Sometimes, however, the orientation specificity at one or more of these sites could not be determined, and they were not used for GABA application. A single cell was isolated with the central electrode, its receptive field mapped and classified as S, C, or B type (Orban, 1984), and a monocular orientation tuning curve for the dominant eye derived. Once a stable control tuning curve had been attained, GABA was applied simultaneously (with the same ejecting current) at sites where the orientation preference was either similar to $\left( \pm 22.5^{\circ}\right.$; "iso-orientation" sites) or markedly different from $\left( \pm 45-90^{\circ}\right.$; "cross-orientation" sites) that of the recorded cell. Comparisons of the orientation preference of cells at the recording and inactivation sites were always made for the eye that was dominant for the recorded cell. The ejecting currents used at "iso-orientation" and "cross-orientation" sites were comparable, and typically in the range of 50-100 nA. Higher currents (maximum $200 \mathrm{nA}$ ) were sometimes used toward the end of the period of GABA iontophoresis if no effects on orientation tuning had been observed. GABA was applied continuously for a maximum of $30 \mathrm{~min}$, during which time orientation tuning curves for the recorded cell were repeatedly derived. Changes in orientation tuning or responsiveness were considered to be related to GABA application only if they were followed by a recovery within 30 min of the termination of iontophoresis. Whenever possible, the effect of inactivating "cross-orientation" and "iso-orientation" sites was compared directly in the same cell. The orientation preference at the inactivation sites was often redetermined, particularly following a significant effect on orientation tuning. In exceptional cases where little or no drive could be elicited at an inactivation site through the eye that was dominant for the recorded cell, GABA was not applied. With this proviso, there was no obvious tendency for the occurrence or magnitude of the effects of "iso-orientation" or "cross-orientation" inactivation to depend on the closeness of the ocular dominance at the recording and inactivation sites, and this aspect is not considered further in Results. Occasionally, a single pipette, rather than the array of four pipettes, was used for inactivation, but in all other respects the procedure was the same.

Except that in some cells the effect of remote inactivation was tested on orientation tuning for a stationary flash-presented bar, rotated symmetrically about the receptive field center (for C-and B-cells) or about the dominant $O N$ or OFF receptive field subregion (for S-cells), the orientation tuning of a recorded cell and of multiunit clusters at the inactivation sites was determined using the same procedure: a light bar of optimal length and width was swept at the optimal velocity in opposite directions along the axis orthogonal to its orientation, with the stimulus sweep centered on the receptive field. Orientation was varied in pseudorandom sequence by multiples of $22.5^{\circ}$; the starting orientation could be adjusted such that the testing orientations always included the optimum determined manually and the orientation orthogonal to it. Perior poststimulus time histograms (PSTHs) were compiled from five responses at each testing orientation (bin width, $10 \mathrm{msec}$ ). To derive polar diagrams, the PSTHs were smoothed by combining an appropriate number of bins (range, 2-10); the peak response from smoothed PSTHs was plotted as vector length, and direction of motion (for moving stimuli) or orientation (for stationary stimuli) as vector angle. Mean spontaneous activity was calculated from each period preceding stimulus motion or stimulus onset at each testing orientation. To exclude the possibility

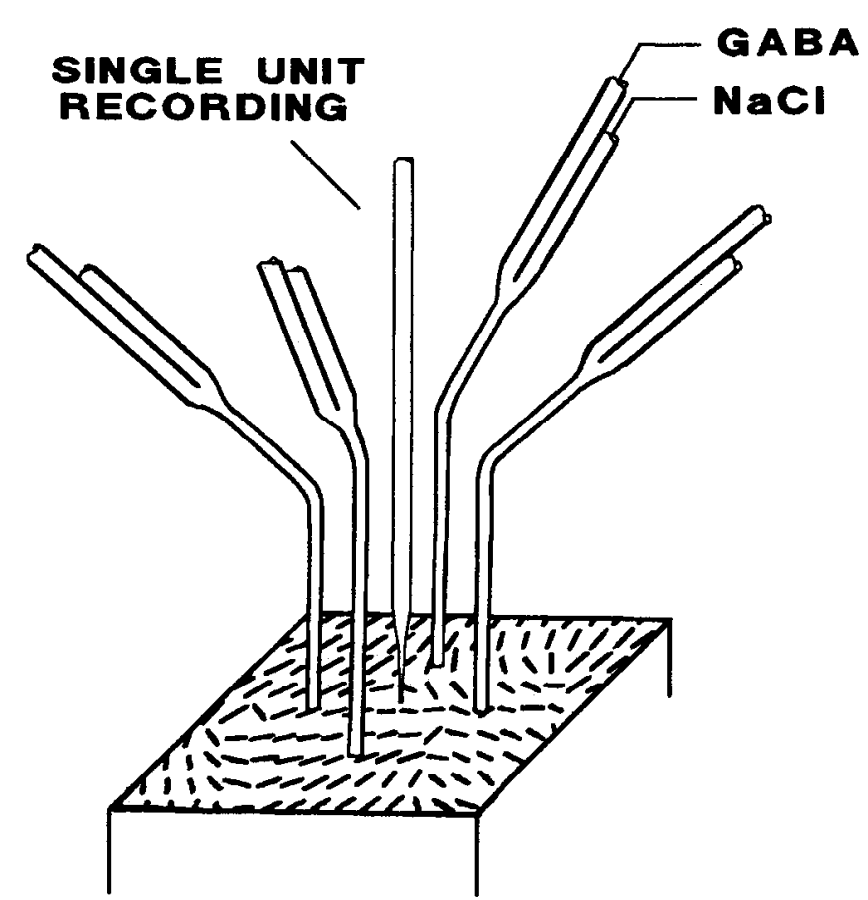

Figure 1. Schematic drawing, in perspective, of the array of four inactivation pipettes and the electrode for single-unit recording, superimposed on the layout of orientation in a small region of area 18. The map of preferred orientation is modified from Swindale et al. (1987). The horizontal separation between the recording and inactivation sites was approximately $600 \mu \mathrm{m}$. At this distance, inactivation sites with radically different orientation preferences from that of a recorded cell were preferentially sampled.

that saturation of response to the optimum orientation contributed to broadening of tuning, stimulus contrast $(0.3-0.5)$ was adjusted to yield clearly submaximal responses in the control situation, and when the response to the optimum orientation increased during remote inactivation, it was verified that this response was not saturated.

To quantify the effects on orientation tuning, the procedure described in detail elsewhere (Crook et al., 1991a) was adopted, whereby orientation tuning width in degrees (measured at half the maximum response) and the relative response to the orientation orthogonal to the optimum (compared with the response to the optimum) were assessed from smoothed tuning curves derived in the control situation and when the effects of GABA application were at their height. Comparisons were made for the preferred direction of motion, or for the dominant $O N$ or OFF response to stationary stimuli. Tuning width was assigned the maximum value of $180^{\circ}$ if the response never fell below half-maximum. If this was the case for responses on only one side of the optimum, halfwidth of tuning was assigned the maximum value of $90^{\circ}$, and tuning width equalled the sum of the two half-widths. The criterion for a significant effect was a change in tuning width of more than $25 \%$ (Crook et al., 1991a), except in four cells that showed a marked increase in response only to the orientation orthogonal to the optimum, with no significant change in measured tuning width.

\section{Results \\ Differential effects of inactivating "cross-orientation" and "iso-orientation" sites}

This report is based on results from 66 single cells with parafoveal receptive fields (4-12 $2^{\circ}$ eccentricity) of the $\mathrm{S}(n=38), \mathrm{C}$ $(n=20)$, or B type $(n=8)$, in which the effect of inactivating "cross-orientation" sites only (44 cells), "iso-orientation" sites only ( 9 cells), or "cross-orientation" and "iso-orientation" sites alternately (13 cells) was successfully tested. The bias in favor of "cross-orientation" sites at a distancc of $600 \mu \mathrm{m}$ reflected the layout of orientation in area 18 (Swindale et al., 1987; see Fig. 
CONTROL

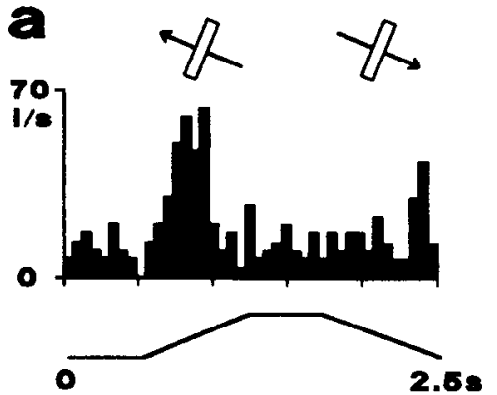

INACTIVATION SITE II
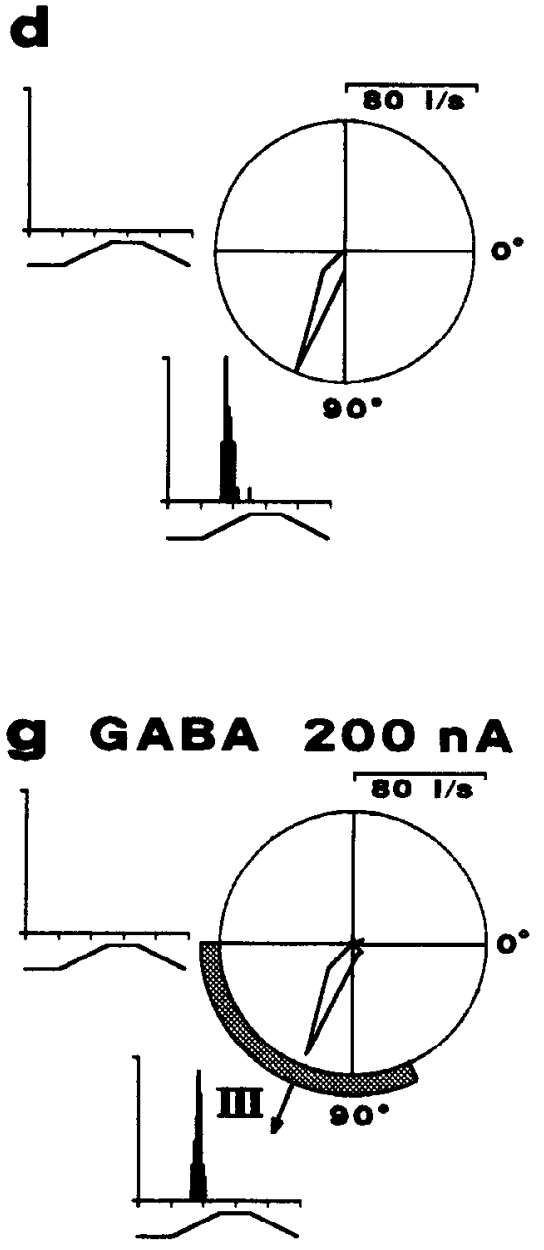

18-20 min
GABA 50 nA
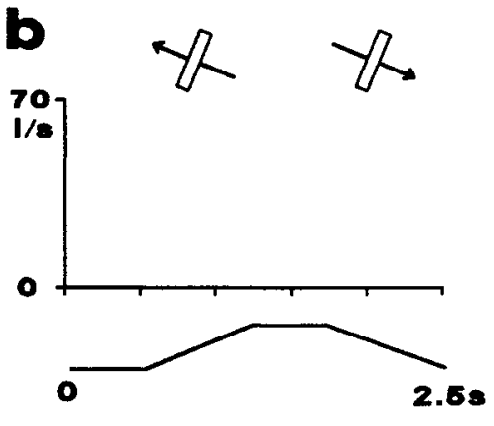

$3 \mathrm{~min}$

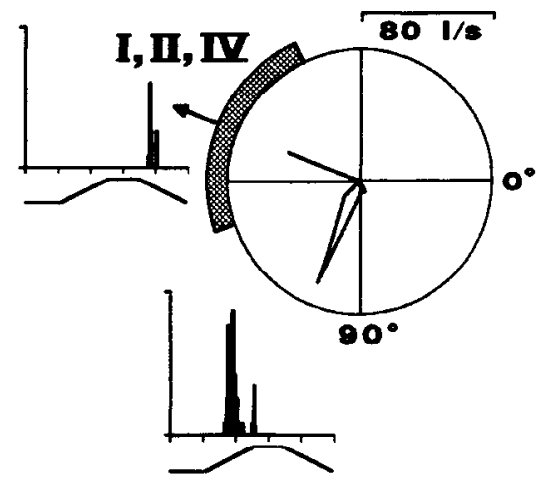

S-7 min
RECOVERY

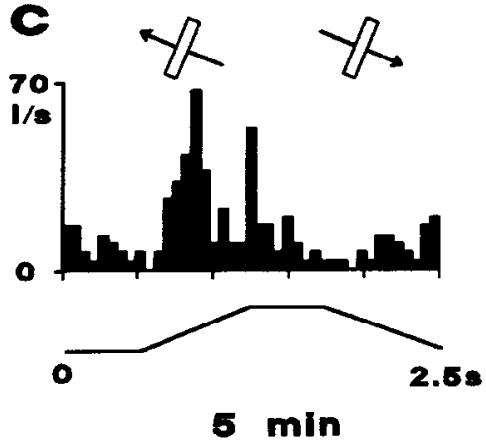

$f$

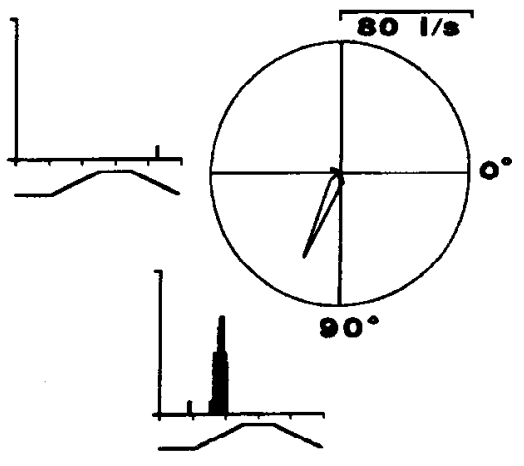

5-7 min

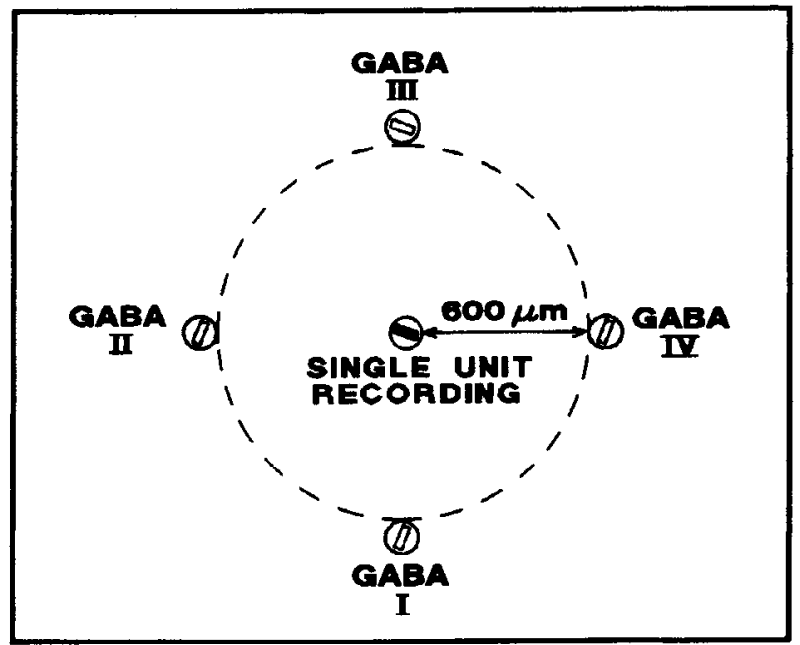

Figure 2. Inset (lower right), Orientation preference of an orientation-selective S-cell (solid bar), and of multiunit clusters at the surrounding sites for GABA application (open bars) at a horizontal distance of some $600 \mu \mathrm{m}$. $a-c$, Reversible inactivation of one of these sites (II) that was used in e. $a$, Multiunit activity recorded via the GABA-containing pipette, in response to back-and-forth motion of an optimally oriented bar. $b$, Abolition of visually driven and spontaneous activity within $3 \mathrm{~min}$ of the onset of continuous iontophoresis of GABA (50 nA) via a second barrel of the same pipette. $c$, Recovery within $5 \mathrm{~min}$ of the termination of GABA application. Direction of motion for an optimally oriented bar is indicated above each PSTH. Stimulus waveform and cycle duration are indicated below each PSTH (five consecutive stimulus cycles); bin width, 60 msec (six bins combined). $d-f$, Appearance of a phasic response to the orientation orthogonal to the optimum in the recorded S-cell during the inactivation of multiple "cross-orientation" sites. $d$, Orientation tuning prior to GABA application. $e$, Tuning assessed 5-7 min after the onset of continuous iontophoresis of GABA $(50 \mathrm{nA})$, simultaneously at three sites $(I, I I, I V)$ where the orientation preference was orthogonal to that of the recorded cell (see inset). $f$, Orientation tuning assessed 5-7 min following termination of GABA application. $g$, Tuning of the same cell following recovery in $f$, and 18-20 min after the onset of continuous iontophoresis of GABA at the remaining site (III), where the orientation preference matched that of the recorded cell. Ejecting current, initially $50 \mathrm{nA}$, was increased during the period of iontophoresis to $200 \mathrm{nA}$. In $e$ and $g$, the arrows indicate the preferred direction at each inactivation site, for motion of an optimally oriented bar, and the shaded area indicates the range of effective orientations; in this and all subsequent examples, these values were derived from orientation tuning curves for the eye that was dominant for the recorded cell. In $d-g$, PSTHs in response to the preferred orientation and the orientation orthogonal to it are placed at the appropriate vector angle around the perimeter of each polar diagram; bin width, $30 \mathrm{msec}$ (three bins combined); vertical calibration, $80 \mathrm{impulses} / \mathrm{sec}$; stimulus waveform indicated below each PSTH; duration of stimulus cycle, $2.5 \mathrm{sec}$; spontaneous activity essentially zero throughout. 


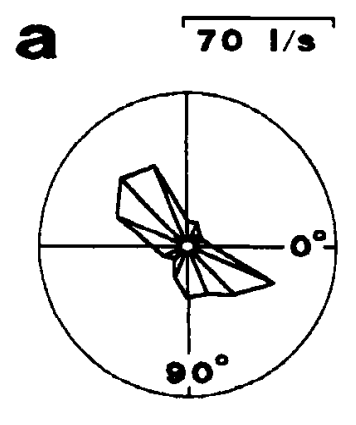

CONTROL

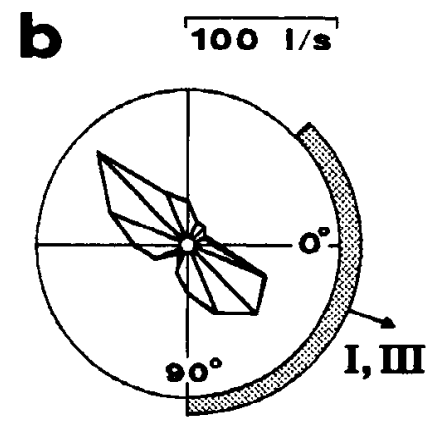

GABA 50 nA

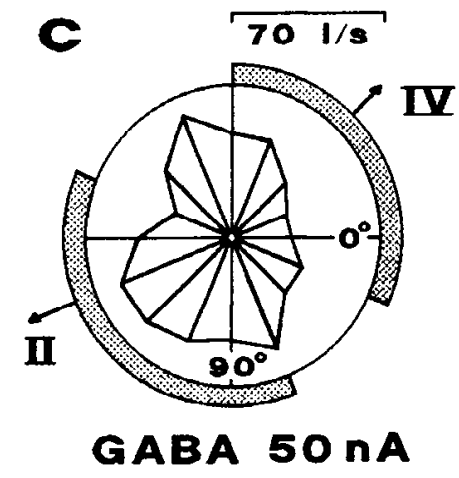

Figure 3. Alternate inactivation of "iso-orientation" and "cross-orientation" sites causes differential effects on the orientation tuning of a C-cell for a moving bar. $a$, Orientation tuning prior to GABA application. $b$, Tuning assessed during simultaneous application of GABA at two sites $(I, I I I)$ where the orientation preference was similar to that of the recorded cell. $c$, Tuning assessed during subsequent application of GABA, simultaneously at two different sites $(I I$ and $I V)$ where the preferred orientations were approximately orthogonal to that of the recorded cell; GABA iontophoresis began $5 \mathrm{~min}$ after the termination of GABA application at sites $I$ and $I I I$. Conventions as in Figure $2 d-g$; additionally, mean spontaneous activity is indicated by the radius of the circle in the center of each polar diagram. Note that the calibration in $b$ is different from that in $a$ and $c$.

1). The receptive fields of cells at the recording and inactivation sites overlapped extensively, but substantial modifications to orientation tuning occurred only during the inactivation of "crossorientation" sites. This is illustrated in Figures 2 and 3, which show extreme examples of the effect of inactivating "cross-orientation" sites.

The inset to Figure 2 indicates the orientation preference of multiunit clusters recorded at each of four inactivation sites, and of an orientation-selective S-cell recorded with the central electrode. The upper row shows multiunit activity recorded at one of the "cross-orientation" inactivation sites, in response to motion of an optimally oriented bar $(a)$, the abolition of visually driven and spontaneous activity during GABA iontophoresis (b), and a recovery following termination of GABA application (c). Reversible abolition of multiunit activity at the other sites (not shown) had a similar time course. Figure $2 d-f$ shows the effect of simultaneous GABA application at three sites where, in each case, the preferred orientation was orthogonal to that of the recorded S-cell. A clear response to this orientation appeared during GABA iontophoresis $(e)$ and disappeared following termination of GABA application $(f)$. This was a consistent phasic response that occurred slightly before the bar crossed the center of the receptive field. This type of response to the orientation orthogonal to the optimum has been observed in S-cells during blockade of GABA-mediated inhibition by iontophoretic application of bicuculline (Sillito, 1975). The relatively narrow profile of the response to the orientation orthogonal to the optimum that appeared during GABA application was typical, although a specific increase in response to this orientation was seen in only a small minority of cells (see below). The time course of the effect on orientation tuning was similar to that for the abolition of multiunit activity recorded at the inactivation sites (compare $e$ with $b$ ). Prolonged GABA application at the remaining site, where the orientation preference matched that of the recorded cell $(g)$, had no significant effect on orientation tuning.

Figure 3 shows another example of a dramatic effect on orientation tuning that occurred only during the inactivation of "cross-orientation" sites. Application of GABA simultaneously at two sites where the orientation preference was similar to that of a $\mathrm{C}$-cell recorded with the central electrode $(b)$ caused no broadening of orientation tuning. The only effect was a marked increase in both response magnitude and spontaneous activity (note the different calibration in $a$ and $b$ ). In contrast, application of GABA simultaneously at two different sites where the preferred orientations were approximately orthogonal to that of the recorded cell $(c)$ caused a marked increase in response to nonoptimal orientations and an elimination of orientation tuning.

Typically, however, the effect of inactivating "cross-orientation" sites consisted in a reduction rather than a loss of orientation tuning. This is documented for two sharply tuned S-cells in Figures 4 and 5 . The bottom row of Figure 4 shows reversible broadening of orientation tuning for a moving bar during simultaneous application of GABA at three sites where the preferred orientations differed by $45^{\circ}$ or more from that of the recorded cell. Again, broadening of tuning was due to an increase in response to nonoptimal orientations and showed a similar time course to that for the abolition of multiunit activity recorded at the inactivation sites (compare $e$ with $b$ ). The orientation specificity at the remaining site could not be determined, and GABA was not applied.

As shown in Figure 5, comparable effects on orientation tuning could be elicited by inactivating a single "cross-orientation" site, and by using a stationary bar rather than a moving bar. In this case, the orientation tuning of the recorded cell was assessed with a flash-presented bar, rotated symmetrically about the dominant OFF subregion of the receptive field. The preferred orientation at the inactivation site, which is indicated by the extended horizontal line at $0^{\circ}$ in $b$, was orthogonal to that of the recorded cell. During GABA application, orientation tuning broadened substantially, due to a selective increase in response to nonoptimal orientations; the cell now gave a significant response to the orientation orthogonal to the optimum. The effect was reversible $(c)$, and its time course characteristic.

\section{Quantitative comparison of the effects of inactivating "cross- orientation" and "iso-orientation" sites}

A quantitative comparison of the effects on orientation tuning elicited by inactivating "cross-orientation" and "iso-orientation" sites is presented in Table 1 and Figure 6 . It can be seen that substantial modifications to orientation tuning occurred only during the inactivation of "cross-orientation" sites, and that the effects typically consisted in a reduction rather than a loss of tuning. In four cells, the significant effect on orientation tuning consisted in a specific increase in response to the orientation orthogonal to the optimum during the inactivation of 
CONTROL

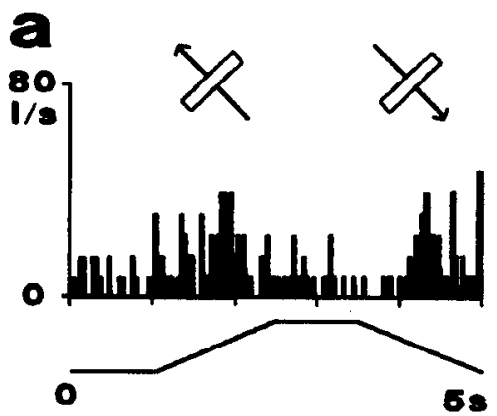

INACTIVATION SITE II
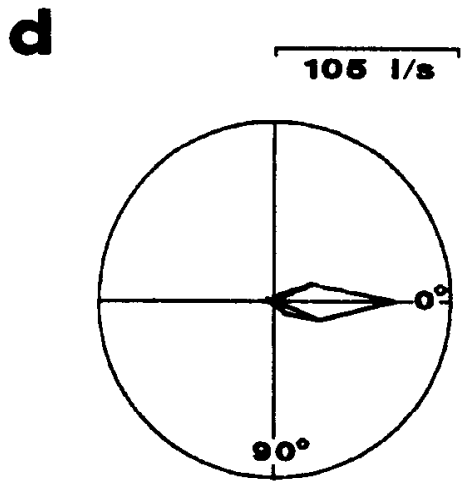

GABA 100 nA

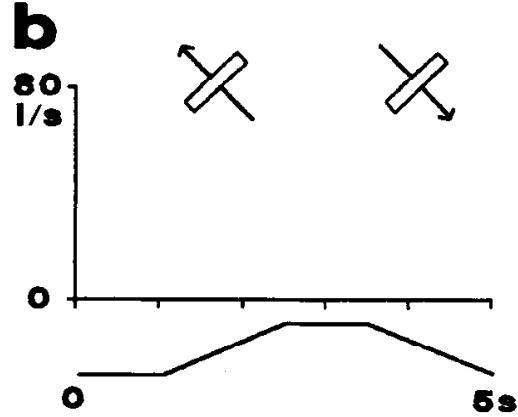

$3 \mathrm{~min}$
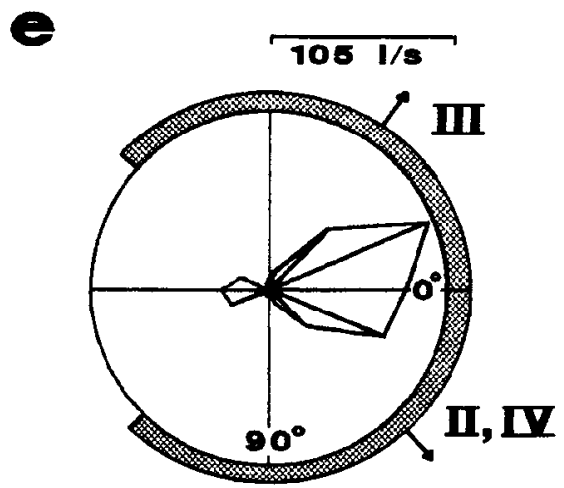

6-8 min
RECOVERY

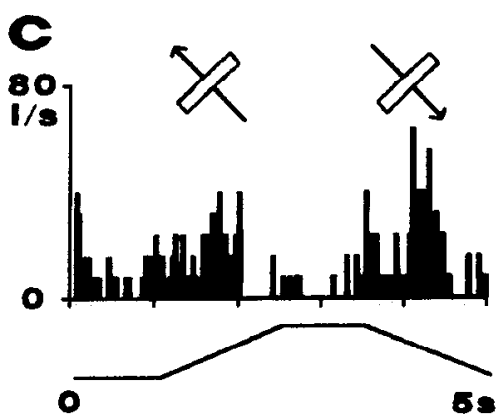

$6 \mathrm{~min}$

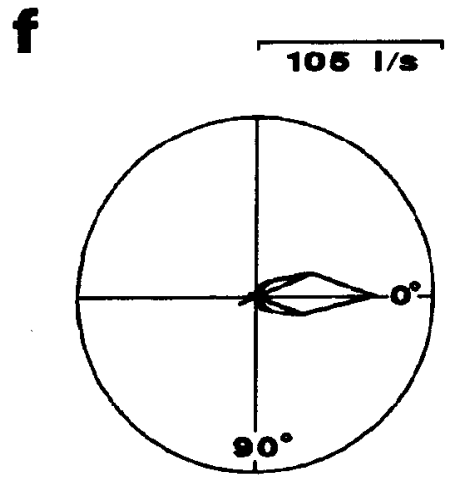

5-7 min

Figure 4. $a-c$, Reversible abolition of multiunit activity recorded at one of the inactivation sites $(I I)$ used in $e$. Conventions and derivation are as in Figure $2 a-c$. PSTH bin width, $40 \mathrm{msec}$ (four bins combined). $d-f$. Reversible broadening of orientation tuning for a moving bar, in an orientation-selective S-cell, induced by inactivating multiple "cross-orientation" sites. $d$, Orientation tuning prior to GABA application. $e$, Tuning assessed 6-8 min after the onset of continuous iontophoresis of GABA, simultaneously at three sites $(I I, I I I, I V)$ where the preferred orientations differed by $45^{\circ}$ or more from that of the recorded cell. $f$. Orientation tuning 5-7 min following termination of GABA application. Conventions are as in Figure $2 d-g$, except that in $e$, the arrow referring to site $I I$ indicates one of two equally effective directions of motion for an optimally oriented bar. Spontaneous activity in $d, e$, and $f$, is $0.6,1.7$, and 0.8 impulses/sec, respectively.

a

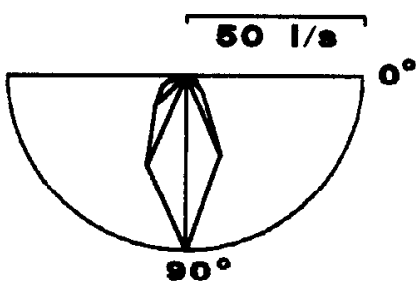

CONTROL b

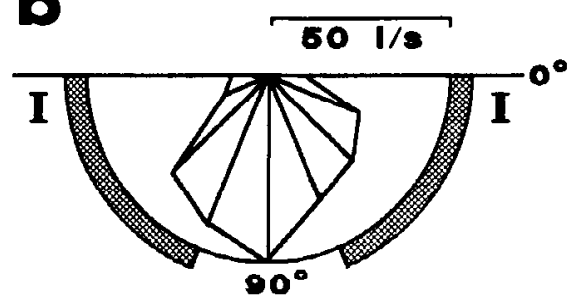

GABA 50 nA 9-11 min
6

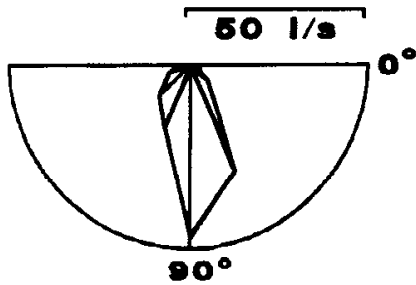

\section{RECOVERY} 6-8 min

Figure 5. Reversible broadening of orientation tuning for a stationary bar, in an orientation-selective S-cell, induced by inactivating a single "cross-orientation" site. The bar was rotated symmetrically about the dominant OFF subregion of the receptive field and flashed on for 2 sec every $5 \mathrm{sec}$ at each testing orientation. The OFF responses are plotted as vector length and orientation as vector angle, $a$, Orientation tuning prior to GABA application. $b$, Tuning assessed 9-11 min after the onset of continuous iontophoresis of GABA at a single site where the preferred orientation was orthogonal to that of the recorded cell. The preferred orientation at the inactivation site (assessed with a moving bar) is indicated by the extended horizontal line at $0^{\circ}$; the range of effective orientations is indicated by the shaded area. $c$, Orientation tuning 6-8 min following termination of GABA application. Spontaneous activity in $a, b$, and $c$ is $0.7,0.9$, and 0.8 impulses $/ \mathrm{sec}$, respectively. 


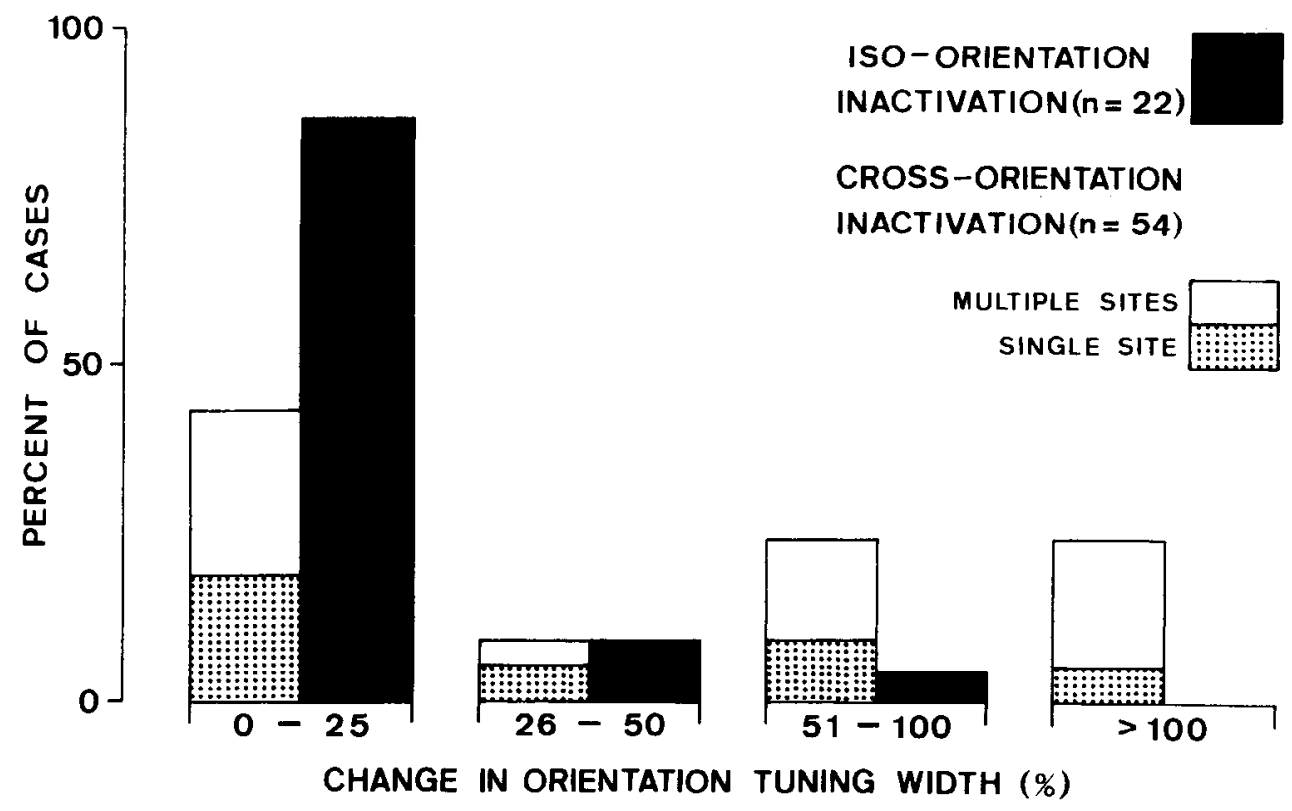

Figure 6. Distribution of the percentage change in orientation tuning width that occurred during the inactivation of "cross-orientation" sites and "iso-orientation" sites. A change of more than $25 \%$ always represented an increase in tuning width. Total histogram height indicates the percentage of "cross-orientation" or "iso-oricntation" inactivations that produced the changes in tuning width shown. Within each histogram column referring to the inactivation of "cross-orientation" sites, the stippled and open areas indicate the proportion of single-site and multiplesite inactivations; results for a single site include cases when a single pipette was used for GABA application, and when the array of four pipettes was used, but only one "cross-orientation" site was inactivated. "cross-orientation" sites, with no change in measured tuning width (Fig. 2). Such effects are reflected in comparisons of the 90:0 $0^{\circ}$ ratio in Table 1 . The difference in the effects on orientation tuning width elicited by inactivating "cross-orientation" and "iso-orientation" sites was highly statistically significant (MannWhitney $U$ test, $p<0.001$ ). Moreover, of the 13 cells in which the effect of applying GABA at "cross-orientation" and "isoorientation" sites was compared directly, 10 showed significant modifications to orientation tuning only during the inactivation of "cross-orientation" sites (Figs. 2, 3). The orientation tuning of the remaining three cells was unaffected by remote inactivation. The difference between $\mathrm{S}$-cells and $\mathrm{C}$-cells in the magnitude of the effects elicited by inactivating "cross-oricntation" sites (shown in Table 1) was not statistically significant, although $C$-cells with only a weak orientation bias were conspicuous in showing no significant effects on orientation tuning.
Table 1 and Figure 6 also compare the effect on orientation tuning of inactivating a single "cross-orientation" site with that elicited by simultaneously inactivating multiple "cross-orientation" sites. On average, simultaneous inactivation of multiple "cross-orientation" sites induced more potent effects on orientation tuning than the inactivation of a single "cross-orientation" site. Additionally, the most radical modifications to orientation tuning usually occurred during the inactivation of multiple "cross-orientation" sites; all cells that lost their orientation tuning and most of those that showed an increase in tuning width of more than $100 \%$ were subjected to inactivation of multiple "cross-orientation" sites. Simultaneous application of GABA at multiple sites not only increased the probability of silencing those cells that contributed to the orientation selectivity of the recorded cell, but also typically influenced a wider range of orientations.

\begin{tabular}{|c|c|c|c|c|c|c|}
\hline & \multirow[b]{2}{*}{$n$} & \multirow{2}{*}{$\begin{array}{l}\text { Significant } \\
\text { effect }\end{array}$} & \multirow{2}{*}{$\begin{array}{l}\text { Increase in } \\
\text { tuning width } \\
(\%)\end{array}$} & \multirow{2}{*}{$\begin{array}{l}\text { Loss of } \\
\text { tuning }\end{array}$} & \multicolumn{2}{|c|}{ 90:0 Ratio } \\
\hline & & & & & Control & GABA \\
\hline Iso-orientation & 22 & $3(14 \%)$ & $39( \pm 13)$ & - & 0.03 & 0.06 \\
\hline Cross-orientation & 54 & $35(65 \%)$ & $93( \pm 55)$ & 4 & 0.07 & 0.27 \\
\hline S-cells & 30 & $21(70 \%)$ & $101( \pm 55)$ & 3 & 0.05 & 0.26 \\
\hline C-cells & 17 & $9(53 \%)$ & $81( \pm 52)$ & 1 & 0.10 & 0.29 \\
\hline Multiple sites & 33 & $22(67 \%)$ & $106( \pm 59)$ & 4 & 0.10 & 0.35 \\
\hline Single site & 21 & $13(62 \%)$ & $73( \pm 42)$ & - & 0.06 & 0.15 \\
\hline
\end{tabular}

Comparisons are for orientation tuning in the control situation and when the effects of GABA application were at their height. The $90: 0^{\circ}$ ratio is the relative response to the orientation orthogonal to the optimum, compared with the response to the optimum. A significant effect on orientation tuning represented an increase in tuning width of more than $25 \%$, except in four cells that showed a marked increase in response only to the orientation orthogonal to the optimum during the inactivation of "cross-orientation" sites. The increase in tuning width and comparisons of the $90: 0^{\circ}$ ratio refer only to cells in which the effects on orientation tuning were significant. The increases in tuning width are means, with SD in parentheses. Results for the inactivation of "cross-orientation" sites are compared for S-cells and C-cells; the remaining cells were B cells. The effects of inactivating a single "cross-orientation" site and of simultaneously inactivating multiple "cross-orientation" sites are shown separately; results for a single "cross-orientation" site include cases when a single pipette was used for GABA application, and when the array of four pipcttes were used, but only one "cross-orientation" site was inactivated. 
Figure 7. Comparison of the percentage change in the response to the optimum orientation that occurred during the inactivation of "cross-orientation" and "iso-orientation" sites; the "crossorientation" population includes only cells that showed a significant effect on orientation tuning. A change of more that $25 \%$ always reflected an increase in response. Results are for the preferred direction of motion or, when stationary stimuli were used, for the dominant ON or OFF response.

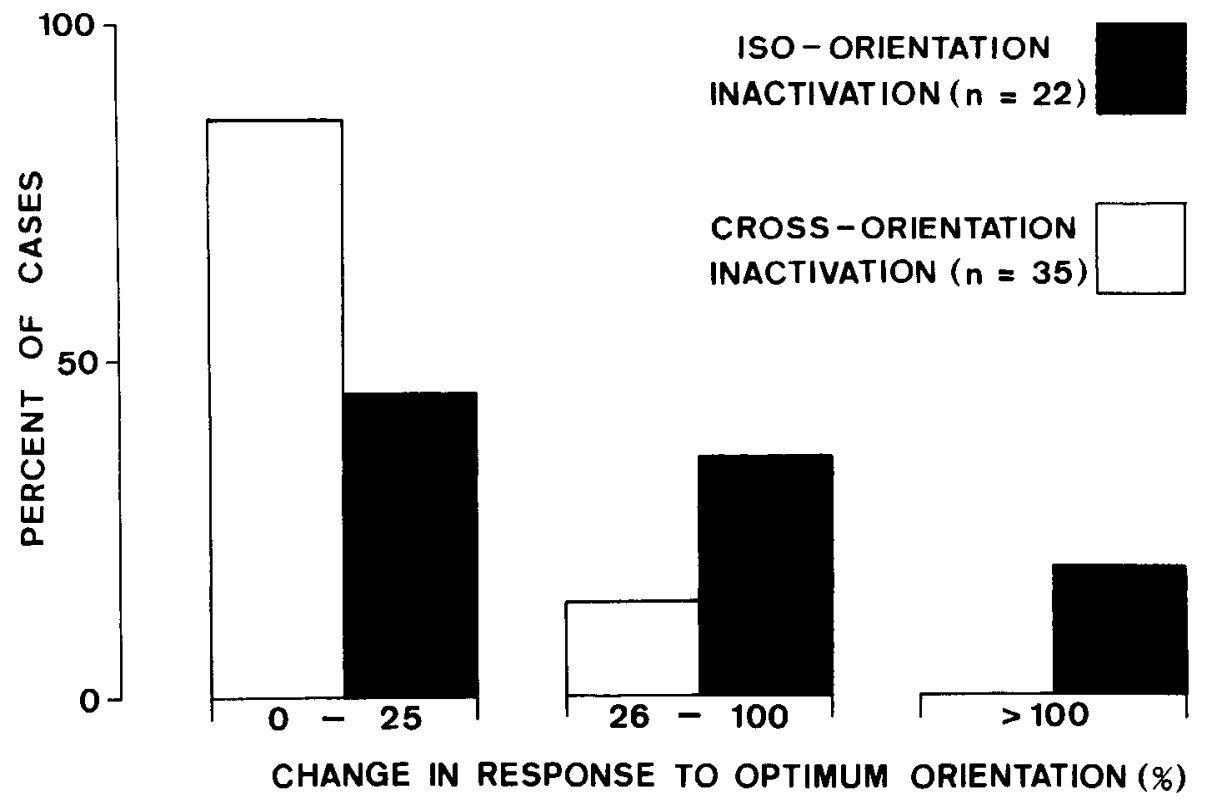

In an attempt to elicit maximal effects on orientation tuning, GABA was applied simultaneously at all available "cross-orientation" or "iso-orientation" sites, and when the array of inactivation pipettes was used, it was usually the case that more "cross-orientation" than "iso-orientation" sites were available for inactivation. Thus, the difference in the effects on orientation tuning elicited by "cross-orientation" and "iso-orientation" inactivations might have been related not to the orientation preference at the inactivation sites, but to the greater number of "cross-orientation" sites that were simultaneously inactivated. This possibility can be excluded for two reasons. First, the difference in the effect of inactivating "cross-orientation" and "iso-orientation" sites was still statistically significant (MannWhitney $U$ test, $p<0.05$ ) when only cases where a single "crossorientation" site had been inactivated were considered (see Table 1). Second, differential effects on orientation tuning could be elicited in the same cell by inactivating the same number of "cross-orientation" and "iso-orientation" sites (Fig. 3).

A further point is that, in many cases, inactivation of "isoorientation" sites did have an effect on orientation tuning curves, but this typically consisted in an increase in response magnitude, with no significant change in tuning width. A comparison of the change in response to the optimum orientation that occurred during the inactivation of "cross-orientation" and "iso-orientation" sites is shown in Figure 7, in which it can be seen that significant modifications to orientation tuning due to the inactivation of "cross-orientation" sites were only rarely accompanied by a significant $(>25 \%)$ change in response (maximum increase, $63 \%$ ). In contrast, during the inactivation of "iso-orientation" sites many cells, including those whose orientation tuning broadened significantly, showed a marked increase in response, with a maximum of $131 \%$. This difference in the effect of inactivating "cross-orientation" and "iso-orientation" sites was statistically significant (Mann-Whitney $U$ test, $p<0.05$ ). Furthermore, in the same cell, inactivation of "cross-orientation" and "iso-orientation" sites could elicit specific effects on orientation tuning and response magnitude, respectively (Fig. 3). In none of the cells that showed an increase in response magnitude during "iso-orientation" inactivation had qualitative testing revealed end-inhibition in the control situation, and it seems that end-stopped cells can be reliably discriminated using qualitative techniques (Kato et al., 1978). Thus, the observed increase in response is unlikely to have been due to a reduction in end-inhibition, and an alternative possibility is discussed below.

\section{Discussion}

The present study has demonstrated that GABA-induced inactivation of multiunit clusters whose receptive fields overlap extensively with that of a recorded cell, but whosc preferred orientations are radically different, can cause the orientation tuning of the recorded cell to broaden substantially. The effects on orientation tuning had a time course similar to that for the abolition of multiunit activity at the inactivation sites, and they could not be replicated by applying GABA at sites where the orientation preference was similar to that of the recorded cell. Thus, the observed broadening of tuning was due to the localized inactivation of cells whose preferred orientations were radically different from that of the recorded cell.

\section{Methodological considerations}

Since the reliable determination of orientation preference at the inactivation sites is crucial to the interpretation of the results, it is important to consider three potential sources of error. First, at least in area 17, a small number of cells within an orientation "column" have preferred orientations that differ radically from that of the "column" optimum (Lee et al., 1977). Therefore, the orientation specificity at the inactivation sites was always assessed on the basis of multiunit responses, since they provided a more reliable estimate of the total local activity influenced by iontophoretic application of GABA. The second potential source of error is related to the problem of determining the orientation preference at multiple sites via pipettes that could not be moved independently. Advancement of the fixed array of pipettes to isolate multiunit activity at one of the inactivation sites would render unreliable the orientation preference that had been de- 
termined at the other sites. For this reason, the array was not moved after the orientation preference at the first inactivation site had been determined. A final problem is that cortical instability might have caused a change in the location of the inactivation sites after the orientation preference of each had been determined. Thus, at the time the effects of GABA application on orientation tuning were tested, the orientation preference at the inactivation sites might have differed from that originally assessed. Since penetrations made with the inactivation pipettes were approximately normal to the cortical surface, and since in area 18 there are no systematic shifts in preferred orientation within a "column" (Berman et al., 1987), such an effect would not be expected to cause gross changes in orientation preference. Additionally, the orientation specificity at the inactivation sites was frequently redetermined, particularly following a positive effect on orientation tuning, and found not to have differed significantly from that determined originally. Thus, errors in the assessment of the preferred orientations at the inactivation sites can be largely excluded.

\section{Significance of the differential effects elicited by inactivating "cross-orientation" and "iso-orientation" sites}

A number of potential explanations for the influence of remote GABA application on orientation tuning, which were discussed at length in Crook et al. (1991a), can now be excluded, because they do not predict the differential effects of inactivating "crossorientation" and "iso-orientation" sites. These include facilitation of excitatory inputs at nonoptimal orientations due to GABA-induced depolarization of the dendrites (Connors et al., 1988) of the recorded cell, and a general increase in cell excitability, coupled with nonlinear interactions between excitatory and inhibitory inputs. With respect to the latter possibility, it is also worth emphasizing that broadening of tuning during the inactivation of "cross-orientation" sites was rarely accompanied by a marked increase in response, while the inactivation of "isooricntation" sites often caused a marked increase in response, with no concomitant broadening of tuning (Fig. 7). Finally, if the high density of $\mathrm{GABA}_{\mathrm{A}}$ receptors on putative inhibitory neurons (Somogyi et al., 1989) makes them more sensitive than excitatory neurons to GABA, then GABA application might have created a transient period of general disinhibition, which in turn might have caused the orientation tuning of the recorded cell to broaden. The potentially greater susceptibility of inhibitory neurons to the inwardly diffusing field of GABA may indeed partially explain why inactivating "cross-orientation" sites was effective in broadening orientation tuning. However, the differential effects of inactivating "cross-orientation" and "isoorientation" sites make the loss of an orientation-unspecific inhibitory input an implausible explanation for the observed broadening of tuning.

\section{Underlying circuitry}

We are left with two fundamentally different explanations for the broadening of orientation tuning that occurred during the inactivation of "cross-orientation" sites. The first is that the effects were due to the loss of a "cross-orientation" inhibitory input to the recorded cell that normally sharpens orientation tuning by suppressing responses to nonoptimal orientations. The second is that GABA application released an intracortical excitatory input at nonoptimal orientations that is normally suppressed by the inhibitory mechanism.

Removal of "cross-orientation" inhibition. The most direct explanation for the effects on orientation tuning is that they were due to the inactivation of a monosynaptic "cross-orientation" inhibitory input to the recorded cell. There are certainly tangential inhibitory connections between sites with dissimilar orientation preferences in area 18 that could account for such direct effects (Eysel and Kisvárday, 1991). Moreover, we now have anatomical evidence for direct inhibitory projections to a recording site from "cross-orientation" sites whose inactivation caused broadening of orientation tuning (Crook et al., 1992). Removal of a disynaptic "cross-orientation" inhibitory input, whereby GABA inactivated excitatory connections to inhibitory interneurons close to the recorded cell that normally sharpen its orientation tuning, cannot be excluded, although pyramidal (excitatory) cells make contact mainly with other pyramidal cells in area 18 (LeVay, 1988).

In area 17 , inhibition at markedly nonoptimal orientations has been detected in intracellular recordings (Creutzfeldt et al., 1974), and in extracellular studies in which maintained discharge levels were increased by microiontophoresis of excitatory amino acids (Hess and Murata, 1974; Sillito, 1979; Ramoa et al., 1986). "Cross-orientation" inhibition can also be seen in the orientation tuning curves of many complex cells with high spontaneous activity in areas 17 and 18 (Crook, 1990; Hammond and Pomfrett, 1990). Such suppression of resting discharge by an inappropriately oriented stimulus is more readily observed in genuine orientation tuning curves derived with a stationary flash-presented bar rather than with a moving bar (Crook, unpublished results). These observations, together with the evidence for inhibitory connections between cells/sites with dissimilar orientation preferences in areas 17 and 18 (Hata et al., 1988; Eysel and Kisvárday, 1991), make the failure of a recent intracellular study (Ferster, 1986) to detect "cross-oriented" IPSPs in area 17 puzzling. One possibility is that the inhibition is mainly of the "shunting" type located remotely on distal dendrites and not detectable at the soma; this does not seem to be the case (Douglas et al., 1988; Martin, 1988). There is thus considerable attraction in the hypothesis (Sillito, 1984; Ferster and Koch, 1987) that in most cells "cross-orientation" inhibition merely sharpens an existing orientation bias, for in this case only a small amount of inhibition would be necessary. That orientation tuning typically broadened but was not eliminated during the simultaneous inactivation of multiple "crossorientation" sites is certainly consistent with this interpretation. The residual orientation bias might then reflect that present in the input from the LGN (Vidyasagar and Urbas, 1982), the combined influence of the geniculate input (Hubel and Wiesel, 1962; Ferster, 1987; Chapman et al., 1991), excitatory connections between cells with similar orientation preferences (Ts'o et al., 1986; Gilbert and Wiesel, 1989), or even an "iso-orientation" inhibitory input (Ferster, 1986, 1988). The present results provide little evidence for a contribution of "iso-orientation" inhibition to orientation selectivity, but do not exclude this possibility. "Iso-orientation" inhibition between adjacent simple cells with overlapping receptive field subregions of opposite type (ON or OFF) (Palmer and Davis, 1981; Toyama et al., 1981; Ferster, 1988) could contribute to orientation tuning (Heggelund, 1981; Orban, 1984; Ferster and Koch, 1987; Ferster, 1988) but would not be amenable to study with the present method. By the same token, an oricntation bias in complex cells might be established by "iso-oriented" flanking inhibition from cells in neighboring hypercolumns with laterally displaced receptive fields (Sillito, 1984; Martin, 1988). In the present study, 
however, the combined receptive field of all cells at the inactivation sites usually overlapped completely that of a recorded cell but was larger. Thus, the increase in response to the optimum orientation seen during "iso-orientation" inactivation (Fig. 7) may have been due to the release of an "iso-orientation" inhibitory input that is involved in shaping the directional response along the preferred axis of motion. In the few cases where "iso-orientation" sites with strong direction bias and the same direction preference were simultaneously inactivated, the recorded cell could show an increase in response to the direction that was closest to the preferred direction at the inactivation sites (Crook et al., 1991b). A detailed investigation of the effect of "iso-orientation" inactivation on direction selectivity is now in progress (J. M. Crook, Z. F. Kisvárday, and U. T. Eysel, unpublished results).

Release of intracortical excitation. An alternative explanation for the effects on orientation tuning is that GABA application at "cross-orientation" sites caused disinhibition of an excitatory input to the recorded cell at nonoptimal orientations. This hypothesis rests heavily on the assumption that GABA preferentially silenced inhibitory interneurons (see above). We have already argued, on the basis of the size of the excitatory discharge regions for moving stimuli during remote inactivation, that broadening of tuning is unlikely to be due to the release of an intracortical excitatory input at nonoptimal orientations (Crook et al., 1991a). Furthermore, this explanation would involve extensive excitatory connections between cells with overlapping receptive fields but dissimilar orientation preferences, whereas the weight of evidence to date favors the view that excitatory connections are made predominantly between cells with similar orientation preferences, in both areas 17 and 18 (Ts'o et al., 1986; Gilbert and Wiesel, 1989; Kisvárday and Eysel, 1991). One study (Matsubara et al., 1987; Matsubara, 1988) has provided evidence for long-range excitatory connections between sites with dissimilar orientation preferences in area 18 , but the tracer injections made were too large to provide information about connectivity patterns over the distance between the recording and inactivation sites in the present study. In a comparable study from our laboratory, in which smaller tracer injections were made, connections between sites with dissimilar orientation preferences in area 18 (including those concerned with approximately the same region of visual space) were found to be predominantly inhibitory (Eysel and Kisvárday, 1991). It would indeed be remarkable if extensive excitatory connections between cells with overlapping receptive fields but dissimilar orientation preferences existed simply to be cut off from influence by the inhibitory mechanism.

\section{Conclusion}

The most plausible explanation for the broadening of orientation tuning observed during the inactivation of "cross-orientation" sites is that it reflected the loss of a (direct or indirect) "cross-orientation" inhibitory input to the recorded cell. Thus, it seems that inhibition between cells with overlapping receptive fields but dissimilar orientation preferences sharpens orientation tuning in area 18 of cat visual cortex. Area 18 was chosen for this study mainly for technical reasons, for it afforded placement of the array of pipettes for inactivation in a relatively flat region of cortex whose cells have paracentral receptive fields. However, since we (Crook et al., 1991a) have previously reported comparable broadening of orientation tuning in areas 17 and 18 during GABA application at a horizontal distance of
$500-600 \mu \mathrm{m}$ from a recorded cell (although in these experiments the orientation specificity at the inactivation sites was not known), "cross-orientation" inhibition may be a common mechanism in both areas that serves to sharpen orientation selectivity.

\section{References}

Berman NEJ, Wilkes NE, Payne BR (1987) Organisation of orientation and direction selectivity in areas 17 and 18 of cat cerebral cortex. J Neurophysiol 58:676-699.

Chapman B, Zahs KR, Stryker MP (1991) Relation of cortical cell orientation selectivity to alignment of receptive fields of the geniculocortical afferents that arborize within a single orientation column in ferret visual cortex. J Neurosci 11:1347-1358.

Connors BW, Malenka RC, Silva LR (1988) Two inhibitory postsynaptic potentials, and $\mathrm{GABA}_{A}$ and $\mathrm{GABA}_{\mathrm{B}}$ receptor-mediated responses in neocortex of rat and cat. J Physiol (Lond) 406:443-468.

Creutzfeldt OD, Kuhnt U, Benevento LA (1974) An intracellular analysis of visual cortical neurones to moving stimuli: responses in a cooperative neuronal network. Exp Brain Res 21:251-274.

Crook JM (1990) Directional tuning of cells in area 18 of the feline visual cortex for visual noise, bar and spot stimuli: a comparison with area 17. Exp Brain Res 80:545-561.

Crook JM, Eysel UT (1991a) Modulation of cortical orientation tuning by iontophoretic application of GABA at functionally characterized sites. In: Synapse-Transmission-Modulation (Elsner N, Penzlin H, eds), p 36. Stuttgart: Thieme.

Crook JM, Eysel UT (1991b) Evidence for cross-orientation inhibition in the visual curtex of the anaesthetized cat. J Physiol (Lond) 438: $156 \mathrm{P}$.

Crook JM, Eysel UT, Machemer HF (1991a) Influence of GABAinduced remote inactivation on the orientation tuning of cells in area 18 of feline visual cortex: a comparison with area 17 . Neuroscience 40:1-12.

Crook JM, Kisvárday ZF, Eysel UT (1991b) Reversible inactivation of functionally characterized sites in cat visual cortex: effects on orientation tuning and directional selectivity. Soc Neurosci Abstr 17: 177.

Crook JM, Kisvárday ZF, Eysel UT (1992) Contribution of lateral inhibition to orientation and direction sclectivity in cat visual cortex (area 18): GABA-inactivation combined with injections of $\left[{ }^{3} \mathrm{H}\right]-\mathrm{ni}$ pecotic acid. In: Rhythmogenesis in neurons and networks (Elsner $\mathrm{N}$, Richter D, eds). Stuttgart: Thieme.

Douglas RJ, Martin KAC, Whitteridge D (1988) Selective responses of visual cortical cells do not depend on shunting inhibition. Nature 332:642-644.

Eysel UT, Kisvárday ZF (1991) Specificity of lateral inhibitory connections in cat visual cortex. Soc Neurosci Abstr 17:116.

Eysel UT, Crook JM, Machemer HF (1990) GABA-induced remote inactivation reveals cross-orientation inhibition in the cat striate cortex. Exp Brain Res 80:626-630.

Ferster D (1986) Orientation selectivity of synaptic potentials in neurons of cat primary visual cortex. J Neurosci 6:1284-1301.

Ferster D (1987) Origin of orientation selective EPSPs in simple cells of cat visual cortex. J Neurosci 7:1780-1791.

Ferster D (1988) Spatially opponent excitation and inhibition in simple cells of the cat visual cortex. J Neurosci 8:1172-1180.

Ferster D, Koch C (1987) Neuronal connections underlying orientation selectivity in cat visual cortex. Trends Neurosci 10:487-492.

Gilbert CD, Wiesel TN (1989) Columnar specificity of intrinsic horizontal and corticocortical connections in cat visual cortex. J Neurosci 9:2432-2442.

Hammond P, Pomfrett CJD (1990) Directionality of cat striate cortical neurones: contribution of suppression. Exp Brain Res 81:417-425.

Hata Y, Tsumoto T, Sato H, Hagihara K, Tamura H (1988) Inhibition contributes to orientation selectivity in visual cortex of cat. Nature 335:815-817.

Heggelund $P$ (1981) Receptive field organisation of simple cells in cat striate cortex. Exp Brain Res 42:89-98.

Hess R, Murata K (1974) Effects of glutamate and GABA on specific response properties of neurones in the visual cortex. Exp Brain Res 21:285-297.

Hubel DH, Wiesel TN (1962) Receptive fields, binocular interaction and functional architecture in the cat's visual cortex. J Physiol (Lond) 160:106-154. 
Hubel DH, Wiesel TN (1965) Receptive fields and functional architecture in two non-striate visual areas (18 and 19) of the cat. J Neurophysiol 28:229-289.

Kato H, Bishop PO, Orban GA (1978) Hypercomplex and simple/ complex cell classifications in cat striate cortex. J Neurophysiol 41: $1071-1095$.

Kisvärday ZF, Eysel UT (1992) Cellular organization of reciprocal patchy networks in layer III of cat visual cortex (area 17). Neuroscience 46:275-286.

Lee BB, Albus K, Heggelund P, Hulme MJ, Creutzfeldt OD (1977) The depth distribution of optimal stimulus orientation for neurones in cat area 17. Exp Brain Res 27:301-314.

LeVay $S$ (1988) Patchy intrinsic projections in visual cortex, area 18 of the cat: morphological and immunocytochemical evidence for an excitatory function. J Comp Neurol 269:265-274.

Martin KAC (1988) From single cells to simple circuits in the cerebral cortex. Q J Exp Physiol 73:637-702.

Matsubara A, Cynader MS, Swindale NV (1987) Anatomical properties and physiological correlates of the intrinsic connections in cat area 18. J Neurosci 7:1428-1446.

Matsubara JA (1988) Local, horizontal connections within area 18 of the cat. In: Progress in brain research, Vol 75, Vision within extrageniculo-striate systems (Hicks TP, Benedek G, eds), pp 163-171. Amsterdam: Elsevier.

Morrone MC, Burr DC, Maffei L (1982) Functional implications of cross-orientation inhibition of cortical visual cells. I. Neurophysiological evidence. Proc R Soc Lond [Biol] 216:335-354.

Orban GA (1984) Studies in brain function, Vol 11, Neuronal operations in the visual cortex. Bcrlin: Springer.

Palmer LA, Davis TL (1981) Receptive field structure in cat striate cortex. J Neurophysiol 46:260-276.
Ramoa AS, Shadlen M, Skottun BC, Freeman RD (1986) A comparison of inhibition in orientation and spatial frequency selectivity of cat visual cortex. Nature 321:237-239.

Sillito AM (1975) The contribution of inhibitory mechanisms to the receptive field properties of neurones in the striate cortex of the cat. J Physiol (Lond) 250:305-329.

Sillito AM (1979) Inhibitory mechanisms influencing complex cell orientation selectivity and their modification at high resting discharge levels. J Physiol (Lond) 289:33-53.

Sillito AM (1984) Functional considerations of the operation of GABAergic inhibitory processes in the visual cortex. In: Cerebral cortex, Vol 2 (Jones EG, Peters A, eds), pp 91-117. New York: Plenum.

Somogyi P, Roberts JDB, Gulyas A, Richards JG, De Blas AL (1989) GABA and the synaptic or non-synaptic localization of benzodiazepine/GABA $A_{\mathrm{A}}$ receptor/ $/ \mathrm{Cl}^{-}$channel complex in visual cortex of cat. Soc Neurosci Abstr 15:1397.

Swindale NV, Matsubara JA, Cynader MS (1987) Surface organisation of orientation and direction selectivity in cat area 18 . J Neurosci 7: 1414-1427.

Toyama K, Kimura M, Tanaka K (1981) Organization of cat visual cortex as investigated by cross-correlation technique. J Neurophysiol 46:202-214.

Ts'o DY, Gilbert CD, Wiesel TN (1986) Relationships between horizontal interactions and functional architecture in cat striate cortex as revealed by cross-correlation analysis. J Neurosci 6:1160-1170.

Vidyasagar TR, Urbas JV (1982) Orientation sensitivity of cat LGN neurones with and without inputs from visual cortical areas 17 and 18. Exp Brain Res 46:157-169. 